\title{
GroEL and the maintenance of bacterial endosymbiosis
}

\author{
Mario Ali Fares ${ }^{1}$, Andrés Moya ${ }^{2}$ and Eladio Barrio ${ }^{2}$ \\ ${ }^{1}$ Molecular Evolution and Bioinformatics Laboratory, Biology Department, National University of Ireland, Maynooth, Co. Kildare, \\ Ireland \\ ${ }^{2}$ Institut Cavanilles de Biodiversitat i Biologia Evolutiva and Department of Genetics, Universitat de València, PO Box 2085, \\ 46071 València, Spain
}

\begin{abstract}
Many eukaryotic organisms have symbiotic associations with obligate intracellular bacteria. The clonal transmission of endosymbionts between host generations should lead to the irreversible fixation of slightly deleterious mutations in their non-recombinant genome by genetic drift. However, the stability of endosymbiosis indicates that some mechanism is involved in the amelioration of the effects of these mutations. We propose that the chaperone GroEL was involved in the acquisition of an endosymbiotic lifestyle not only by means of its over-production, as proposed by Moran, but also by its adaptive evolution mediated by positive selection to improve the interaction with the unstable endosymbiont proteome.
\end{abstract}

Although eukaryotes display great diversity and morphological complexity, most of them show limited metabolic capabilities in comparison with prokaryotes, and some have lost the ability to synthesize the essential amino acids and coenzymes that are necessary for their basic metabolism. Some eukaryotes, mainly insects, have solved these limitations by associations with OBLIGATE ENDOSYMBIONTS (see Glossary). The acquisition of an obligate intracellular lifestyle has imposed genetic and physiological transformations to the bacterial partner. Recently, the analysis of large genome sequences from different insect endosymbiotic bacteria has revealed some of the mechanisms that are involved in the transformation of free-living bacteria into obligate bacterial endosymbionts, and their consequences [1].

One consequence of the endosymbiotic lifestyle is the fixation of SLIGHTLY DELETERIOUS MUTATIONS in the nonrecombining endosymbiont genome [2,3]. This fixation is due to the strong effect of GENETIC DRIFT during the strict vertical transmission in the host, from mother to offspring, of a reduced number of endosymbionts imposing a POPULATION BOTTLENECK [4]. The increased MUTATIONAL LOAD over time should lead to a decline in the BIOLOGICAL FITNESS, a phenomenon known as MUlleR's RATCHET [5] and the eventual extinction in the absence of recombination [6,7]. However, the stability of endosymbiosis indicates that compensating mechanisms ameliorate the effect of the

Corresponding author: Eladio Barrio (eladio.barrio@uv.es)

Available online 20 July 2004 mild deleterious genetic variation, enabling endosymbionts to escape from fitness decline.

The bacterial CHAPERONE GroEL, which is involved in the buffering of environmental changes in bacteria, has been postulated as an important protagonist in the acquisition of an endosymbiotic lifestyle by becoming involved in the amelioration of mild deleterious genetic variation in the endosymbiont proteome [2]. In this article, we propose that this new role was achieved not only by an increase in the expression levels of the GroEL-coding gene but also by the action of POSITIVE SELECTION improving the interaction of GroEL with the unstable endosymbiont proteome.

Chaperones as essential surviving-machines for the cell Folding, trafficking and degradation of proteins are processes that depend on the assistance of chaperones, also called heat-shock proteins (Hsp) [8]. Chaperones are

\footnotetext{
Glossary

Adaptive evolution: an evolutionary process that is directed by positive selection, which makes a population better adapted to live in a particular environment.

Biological fitness: the average reproductive success of a genotype in a particular environment relative to other genotypes.

Compensatory mutations: in highly integrated genetic systems, mutations in any one component can have a deleterious effect on fitness; but mutations in several components could lead to a recovery of the fitness reduction or even lead to an overall increase in fitness compared with the current state.

Convergent evolution: process by which a similar character evolves independently from different ancestors (also known as convergence).

Genetic drift: random changes in the gene frequencies. The effect of genetic drift is more effective in small populations than in large populations.

Muller's ratchet: the process by which the number of deleterious mutations increases irreversibly in non-recombinant asexual populations due to the impossibility to recover the less mutated class.

Mutational load: a reduction of the average fitness of the members of a population because of the accumulation of deleterious mutations in the population at any given time.

Obligate endosymbionts: beneficial symbionts that have intracellular lifestyle in specific host cells and depend on the host for survival.

Population bottleneck: a severe reduction in population size, which randomly reduces the genetic variability of a population.

Positive selection: process by which alleles that confer a higher relative fitness to the genotypes increase their frequency and, ultimately, become fixed in the population.

Purifying selection: selection acting against deleterious alleles that arise in a population, preventing their increase in frequency and ensuring their eventual disappearance.

Slightly deleterious mutations: deleterious mutations with such a small selective disadvantage (less than the inverse of twice the effective population size) that their evolutionary fate is influenced more by genetic drift than selection.
} 
present in all eukaryotic and prokaryotic cells where they are expressed constitutively. They are involved in the buffering of environmental changes by increasing their expression under stressful conditions promoted by heat-shock, oxidative stress, nutritional deficiencies, ultraviolet irradiation, chemicals, viral infections, and so on. [8].

The requirement of molecular chaperones to fold the newly synthesized proteins appropriately has also been proven during the past decade $[9,10]$. In contrast to small proteins that are able to fold spontaneously in vitro, multidomain proteins are prone to misfolding and aggregation because of hydrophobic interactions between their unshielded exposed surfaces [11]. Molecular chaperones are the most successful folding machines that cells have developed to prevent protein aggregation. The three major chaperones that are responsible for protein folding are GroEL, Hsp70 and Hsp90. Hsp70, called DnaK in bacteria, is the most ubiquitous and is located in the cytosol and in the cellular compartments of eukaryotic cells and is also found in archaea. By contrast, GroEL, the best-characterized chaperone to date [12-14], is found specifically in bacteria and in the organelles of eukaryotic cells. Hsp90 is a specialized chaperone that is only present in eukaryotic cells.

\section{The high functional flexibility of GroEL}

There is a large body of structural and experimental data demonstrating that GroEL binds to a wide variety of protein conformations and sizes, including intermediates at different folding stages $[15,16]$, completely unfolded proteins [17], molten-globule-like proteins [18], in addition to helical and extended structures [19,20]; therefore, it is difficult to establish a common feature for GroEL protein substrates. Despite the lack of apparent substrate specificity of GroEL, its preference for exposed hydrophobic regions and its flexibility suggest a common mechanism for the interaction with its diverse client proteins [21].

The remarkable ability of GroEL to both fold and unfold proteins has been demonstrated [17,22]. Unfolding of kinetically trapped conformations would enable their degradation or provide the opportunity for productive folding [23].

Given the high flexibility of GroEL (i.e. binding to protein-folding intermediates and halting the non-specific aggregation of unfolded proteins), it is easy to deduce how this chaperone might protect the cell from protein conformations that have accumulated destabilizing amino-acid replacements [24]. The ability of GroEL to ameliorate the effect of mutations is supported by the observation that plasmids that overexpress the operon groE (also called mopBA), which encodes GroEL and its cochaperone GroES, can suppress temperature-sensitive mutations $[25,26]$.

\section{GroEL masks the effects of harmful mutations in the endosymbiotic bacteria of insects}

As mentioned previously, obligate endosymbiotic bacteria are expected to accumulate slightly deleterious mutations as a result of their distinctive population structure. In fact, fast fixation rates of amino-acid substitutions due to genetic drift have been demonstrated in several protein-coding genes from the aphid endosymbiont Buchnera $[2,3]$. The fixation by genetic drift of point mutations in proteincoding genes might produce amino-acid replacements affecting the functional conformation of proteins. In this way, the thermodynamic instability of Buchnera proteins has been demonstrated by a comparison of the normalized energy gap $(\alpha)$ of proteins from endosymbiotic bacteria, pathogenic intracellular bacteria and their free-living relatives [27]. The ability of GroEL to rescue nonproductive protein conformers, which enables multiple chances to advance to the most stable state, provides a compensation mechanism to ameliorate the effects of point mutations inducing loss of protein conformation until compensating mutations accumulate or a new energy minimum is reached. This role, originally proposed by Moran [2], is supported by several observations.

The groE (mopBA) operon is the most highly constitutively expressed loci in insect endosymbiotic bacteria, even during non-stress conditions [28]. Thus, GroEL constitutes 10\% of the total protein in Buchnera [29] and is also overproduced in other endosymbiotic bacteria of different insects [30-33] and Amoeba proteus [34].

During heat-stress conditions, gro $E$ and other heatshock genes show a low transcriptional increase in Buchnera compared with the free-living relative $E$. coli [28]. But this modest stress response fails to rescue heatstressed Buchnera both in laboratory conditions [35] and in nature [36] - because the overproduced GroEL is probably devoted to the rescue of non-functional proteins that are destabilized by the accumulation of slightly deleterious mutations [28].

Finally, in an experiment mimicking the population dynamics of endosymbiotic bacteria [37], the constitutive overexpression of groE is responsible for the significant recovery of biological fitness in Escherichia coli strains that had accumulated deleterious mutations during 3240 generations under strong bottleneck conditions.

However, a comparative analysis of the evolution of the groE operon in endosymbiotic and free-living bacteria showed that the GroEL-encoding gene, in contrast to other endosymbiotic genes, is mainly subjected to PURIFYING SELECTION [38]. The lower frequency of slightly deleterious mutations in groE compared with other endosymbiont genes [39] supports the essential role of this chaperone in the maintenance of the endosymbiont proteome because mutations negatively affecting its function are removed by purifying selection. However, the methods used to analyse GroEL evolution in different endosymbionts also detected specific amino-acid replacements in this chaperone driven by positive selection [38]. Thus, a concentration of positive-selected replacements in key amino-acid positions involved in the interactions between GroEL, GroES and their client proteins was detected, indicating ADAPTIVE EVOLUTION in GroEL, which improves its interaction with the unstable endosymbiont proteome.

We have also tested the hypothesis of CONVERGENT EVOLUTION of GroEL masking the effect of slightly deleterious mutations in endosymbionts belonging to two distantly related bacterial phyla, Proteobacteria and 
Bacteroidetes. Remarkably, the same GroEL aminoacid positions were identified that fix amino-acid substitutions by positive selection in both groups of endosymbiotic bacteria. These positively selected amino-acid variations have occurred in both endosymbiotic groups at the same key amino-acid positions that are involved in the binding of non-native protein conformers and GroES. These results clearly indicate that the deleterious mutation buffering role of GroEL might have been convergently acquired in, at least, two phylogenetically distant bacterial lineages with endosymbiotic lifestyle.

\section{Conclusions}

The function of GroEL should be viewed in the context of the genetic characteristics of the genome of endosymbiotic bacteria. We propose that after the acquisition of an endosymbiotic lifestyle, in such a stable environment, GroEL changed its role from one that buffered the environmental changes to one that led to the amelioration of mild deleterious genetic variation affecting the stability of the endosymbiont proteome, as proposed by Moran [2]. The evolution of this new role was favoured not only by the constitutive overexpression of groE but also by the action of positive selection on those GroEL amino-acid positions that are involved in the interaction with client proteins.

Two groundbreaking publications have also demonstrated that another chaperone, Hsp90, is involved in the stability of mature conformations of signal transducers in eukaryotes. An increase in temperature or the pharmacological inhibition of Hsp90 provided evidence of the high phenotypic variation that is silenced by this chaperone in Drosophila melanogaster [40] and in Arabidopsis thaliana [41]. The buffering capacity might be a universal function of chaperones but, unlike GroEL, eukaryotic chaperones, especially in multicellular organisms, can be more specific in their buffering ability.

Finally, we can not discard the possibility that other mechanisms could act synergistically with GroEL in the maintenance of the intracellular lifestyle. Moreover, COMPENSATORY MUTATIONS fixed in the genome of these bacteria might also reduce the effect of slightly deleterious mutations.

\section{References}

1 Wernegreen, J.J. (2002) Genome evolution in bacterial endosymbionts of insects. Nat. Rev. Genet. 3, 850-861

2 Moran, N.A. (1996) Accelerated evolution and Muller's ratchet in endosymbiotic bacteria. Proc. Natl. Acad. Sci. U. S. A. 93, $2873-2878$

3 Funk, D.J. et al. (2001) Intraespecific variation in symbiont genomes: bottlenecks and the aphid-buchnera association. Genetics 157, 477-489

4 Mira, A. and Moran, N.A. (2002) Estimating population size and transmition bottlenecks in maternally transmitted endosymbiotic bacteria. Microb. Ecol. 44, 137-143

5 Muller, H.J. (1964) The relation of the recombination to mutational advance. Mutat. Res. 106, 2-9

6 Kondrashov, A.S. (1988) Deleterious mutations and the evolution of sexual reproduction. Nature 336, 435-440

7 Lynch, M. et al. (1993) The mutational meltdown in asexual populations. J. Hered. 84, 339-344
8 Hightower, L.E. (1991) Heat shock, stress proteins, chaperones, and proteotoxicity. Cell 66, 191-197

9 Hartl, F.U. (1996) Molecular chaperones in cellular protein folding. Nature 381, 571-579

10 Frydman, J. (2001) Folding of newly translated proteins in vivo: the role of molecular chaperones. Annu. Rev. Biochem. 70, 603-647

11 Buchner, J. (1996) Supervising the fold: functional principles of molecular chaperones. FASEB J. 10, 10-19

12 Sigler, P.B. et al. (1998) Structure and function in GroEL-mediated protein folding. Annu. Rev. Biochem. 67, 581-608

13 Thirumalai, D. and Lorimer, G.H. (2001) Chaperonin-mediated protein folding. Annu. Rev. Biophys. Biomol. Struct. 30, 245-269

14 Grallert, H. and Buchner, J. (2001) A structural view of the GroE chaperone cycle. J. Struct. Biol. 135, 95-103

15 Lilie, H. and Buchner, J. (1995) Interaction of GroEL with a highly structured folding intermediate: iterative binding cycles do not involve unfolding. Proc. Natl. Acad. Sci. U. S. A. 92, $8100-8104$

16 Clark, A.C. and Frieden, C. (1997) GroEL-mediated folding of structurally homologous dihydrofolate reductases. J. Mol. Biol. 268, $512-525$

17 Zahn, R. et al. (1996) Effect of single point mutations in citrate synthase on binding to GroEL. FEBS Lett. 380, 152-156

18 Hayer-Hartl, M.K. et al. (1994) Conformational specificity of the chaperonin GroEL for the compact folding intermediates of alphalactalbumin. EMBO J. 13, 3192-3202

19 Landry, S.J. et al. (1992) Different conformations for the same polypeptide bound to chaperones DnaK and GroEL. Nature 355, $455-457$

20 Buckle, A.M. et al. (1997) A structural model for GroEL-polypeptide recognition. Proc. Natl. Acad. Sci. U. S. A. 94, 3571-3575

21 Braig, K. et al. (1995) Conformational variability in the refined structure of the chaperonin GroEL at $2.8 \AA$ resolution. Nat. Struct. Biol. 2, 1083-1094

22 Zahn, R. et al. (1994) Destabilization of the complete protein secondary structure on binding to the chaperone GroEL. Nature $368,261-265$

23 Kandror, O. et al. (1994) Rapid degradation of an abnormal protein in Escherichia coli involves the chaperones GroEL and GroES. J. Biol. Chem. 269, 23575-23582

24 Todd, M.J. et al. (1996) Chaperonin-facilitated protein folding: optimization of rate and yield by an iterative annealing mechanism. Proc. Natl. Acad. Sci. U. S. A. 93, 4030-4035

25 Van Dyck, T. et al. (1989) Demonstration by genetic suppression of interaction of GroEL products with many proteins. Nature 342, 451-453

26 Gordon, C.L. et al. (1994) Selective in vivo rescue by GroEL/ES of thermolabile folding intermediates to phage P22 structural proteins. J. Biol. Chem. 269, 27941-27951

27 Van Ham, R.C. et al. (2003) Reductive genome evolution in Buchnera aphidicola. Proc. Natl. Acad. Sci. U. S. A. 100, 581-586

28 Wilcox, J.L. et al. (2003) Consequences of reductive evolution for gene expression in an obligate endosymbiont. Mol. Microbiol. 48, 1491-1500

29 Baumann, P. et al. (1996) Levels of Buchnera aphidicola chaperonin GroEL during growth of the aphid Schizaphis graminum. Curr. Microbiol. 32, 279-285

30 Aksoy, S. (1995) Molecular analysis of the endosymbiont of tsetse flies: $16 \mathrm{~S}$ rDNA and over-expression of a chaperonin. Insect Mol. Biol. 4, 23-29

31 Sato, S. and Ishikawa, H. (1997) Expression and control of an operon from an intracellular symbiont which is homologous to the groE operon. J. Bacteriol. 179, 2300-2304

32 Charles, H. et al. (1997) A molecular aspect of symbiotic interactions between the weevil Sitophilus oryzae and its endosymbiotic bacteria: over-expression of a chaperonin. Biochem. Biophys. Res. Commun. $239,769-774$

33 Haines, L.R. et al. (2002) The major protein in the midgut of teneral Glossina morsitans morsitans is a molecular chaperone from the endosymbiotic bacterium Wigglesworthia glossidina. Insect Biochem. Mol. Biol. 32, 1429-1438 
34 Choi, E. et al. (1991) Elevated levels of stress proteins associated with bacterial symbiosis in Amoeba proteus and soybean root nodule cells. BioSystems 25, 205-212

35 Ohtaka, C. and Ishikawa, H. (1991) Effects of heat-treatment on the symbiotic systems of an aphid mycetocyte. Symbiosis 11, 19-30

36 Montllor, C.B. et al. (2002) Facultative bacterial endosymbionts benefit pea aphids Acyrthosiphon pisum under heat stress. Ecol. Entomol. 27, 189-195

37 Fares, M.A. et al. (2002) GroEL buffers against deleterious mutations. Nature 417, 398
38 Fares, M.A. et al. (2002) The evolution of the heat-shock protein GroEL from Buchnera, the primary endosymbiont of aphids, is governed by positive selection. Mol. Biol. Evol. 19, 1162-1170

39 Herbeck, J.T. et al. (2003) A conservative test of genetic drift in the endosymbiotic bacterium Buchnera: slightly deleterious mutations in the chaperonin groEL. Genetics 165, 1651-1660

40 Rutherford, S.L. and Lindquist, S. (1998) Hsp90 as a capacitor of morphologic variation. Nature 396, 336-342

41 Queitsch, C. et al. (2002) Hsp90 as a capacitor of phenotypic variation. Nature 417, 618-623

\title{
Articles of interest in Trends and Current Opinion journals
}

\author{
Professor John Maynard Smith 1920-2004 \\ Sean Nee \\ Trends in Ecology and Evolution, 19, 345-346
}

Bioethics for technology?

Rosie Hails

Current Opinion in Biotechnology, 15, 250-253

Telomere erosion: a new link between HLA DR4 and rheumatoid arthritis?

Mike Salmon and Arne N. Akbar

Trends in Immunology, 25, 339-341

Society and ethics - the genetics of disease

Sandy M. Thomas

Current Opinion in Genetics and Development, 14, 287-291

Evolutionary significance of stress-induced mutagenesis in bacteria

Olivier Tenaillon, Erick Denamur and Ivan Matic

Trends in Microbiology, 12, 264-270

What is genetic quality?

John Hunt, Luc F. Bussière, Michael D. Jennions and Robert Brooks

Trends in Ecology and Evolution, 19, 329-333

RNA repair using spliceosome-mediated RNA trans-splicing

S. Gary Mansfield, Hengjun Chao and Christopher E. Walsh

Trends in Molecular Medicine, 10, 263-268

Dissecting complex behaviours in the post-genomic era

Martien J. H. Kas and Jan M. Van Ree

Trends in Neurosciences, 27, 366-369 